Belastung. $43 \%$ von allen Patienten hatten eine PAH aufgrund einer linksventrikulären Dysfunktion. [1]

Prof. Erich Stoelben, Lungenklinik Köln Merheim, Lehrstuhl für Thoraxchirurgie, Private Universität Witten Herdecke, zufolge ist es prognostisch entscheidend, dass bei Patienten mit respiratorischer Dysfunktion, etwa aufgrund einer COPD, eine eventuell begleitende $\mathrm{PAH}$ rechtzeitig erkannt und behandelt wird. Echokardiografie, kathetergestützte Druckmessungen und CT mit Kontrastmittel seien dabei die entscheidenden diagnostischen Instrumente. „Oft besteht ein komplexes Zusammenspiel zwischen PAH, COPD, Hypoxämie, Entzündungsprozessen und kardialer Funktion“, erklärte Stoelben.

Wenn eine COPD die zugrunde liegende Ursache für die PAH ist, dann sei die Lungenfunktion, so Stoelben, in der Regel nur leicht eingeschränkt und die $\mathrm{PAH}$ somit nur geringgradig, was aber auch dazu beiträgt, dass sie oft übersehen wird. Eine PAH an sich scheint das perioperative Risiko für kardiovaskuläre
Komplikationen und Mortalität bei Lungenresektionen auch noch nicht zu erhöhen. [2] Je schwerer die PAH aber ist - gemessen beispielsweise anhand der Pulmonalarterien-Vergrößerung im CT - desto höher ist das Risiko. [3]

Dr. med. Thomas M. Heim

\section{Literatur}

1. Bründler $\mathrm{H}$ et al. Right heart catheterization in the pre-operative evaluation of patients with lung cancer. Respiration 1985;48:261-68.

2. Wei B et al. The impact of pulmonary hypertension on morbidity and mortality following major lung resection. Eur J Cardiothorac Surg 2014; 45:1028-33.

3. Asakura K et al. Pulmonary arterial enlargement predicts cardiopulmonary complications after pulmonary resection for lung cancer: a retrospective cohort study. J Cardiothorac Surg 2015;10:113.

Quelle: Anästhesiologisch-thoraxchirurgisches Kolloquium am 29.09.2016 im Rahmen der 25. Jahrestagung der Deutschen Gesellschaft für Thoraxchirurgie und 4. D-A-CH-Tagung, Freiburg.

\title{
Günstigere Verläufe nach videoassistiertem thorakoskopischem Zugang?
}

\author{
Trotz Fortschritten in der operativen und antibiotischen Behandlung ist das Pleuraempyem immer noch \\ eine lebensbedrohliche Erkrankung. Eine frühzeitige chirurgische Behandlung über den videoassistier- \\ ten thorakoskopischen (VATS)-Zugang scheint sich positiv auf den Krankheitsverlauf auszuwirken.
}

\author{
Als Pleuraempyem bezeichnet man einen eitrigen Pleuraerguss \\ und unterscheidet drei Stadien: \\ - Stadium I: exsudative Pleuritis \\ - Stadium II: fibrinös-purulentes Pleuraempyem \\ - Stadium III Fibrosierung mit der Bildung von Pleuraschwar- \\ ten und Fibrothorax
}

In den meisten Fällen entsteht das Pleuraempyem parapneumonisch, das heißt auf dem Boden einer bakteriellen Pneumonie, seltener infolge operativer Eingriffe, Thoraxtraumata oder abdomineller Infektionen. Typische Erreger sind Pneumokokken, Staphylococcus aureus, Streptococcus pyogenes und Haemophilus influenzae.

\section{VATS im Vormarsch}

„Als Erstlinientherapie des Pleuraempyems wird neben den Optionen der offenen Thoraxdrainage oder Dekortikation via Thorakotomie zunehmend auch die videoassistierte Thorakoskopie (VATS) eingesetzt“, sagte Dr. Holger Höfken, Thoraxzentrum Ruhrgebiet, Ev. Krankenhaus Herne. Höfken berichtet aus einer Fallserie aus den Jahren 2003 bis 2013 bei Patienten mit parapneumonischem Pleuraempyem, davon 41 im Stadium I, 142 im Stadium II und 65 im Stadium III, die nach einem standardisierten Verfahren von ein- und demselben Team aus fünf Operateuren mittels VATS versorgt wurden. Alle Patienten wurden mit Debridement und Lavage und anschließender diskontinuierlicher täglicher Spülung der Pleurahöhle über fünf Tage behandelt. In Stadium II wurde gegebenenfalls, im
Stadium III immer dekortiziert, eventuell wurde gleichzeitig parietal pleurektomiert. Wenn laut präoperativer CT noch eine Pneumonie bestand, wurde flankierend antibiotisch behandelt.

\section{Hohe Sanierungsraten}

In der von Höfken präsentierten Fallstudie betrug die Erfolgsquote $100 \%$ im Stadium I, $80 \%$ im Stadium II und $63 \%$ im Stadium III. Im Stadium II mussten $25 \%$ der Fälle revidiert werden, davon $76 \%$ per VATS und $24 \%$ per Thorakotomie. Bei allen Patienten konnten damit eine sekundäre Sanierung erreicht werden. In Stadium III benötigte die Hälfte der Patienten eine Revision per VATS, mit einer sekundären Sanierungsrate von 69\%. „Patienten unter 55 Jahren hatten eine längere Krankheitsdauer und ein höheres Empyemstadium“, ergänzte Höfken. Mit Verweis auf gängige Standards und auf Erfahrungswerte schloss Höfken, dass man mithilfe der VATS die Rate der Konversionen zur offenen OP insbesondere in höheren Stadien senken könne. Das sei auch bei Patienten mit kardialer und renaler Komorbidität von besonderer Bedeutung, denn diese hätten einen ungünstigen Einfluss auf das Überleben gezeigt. „Die Daten sprechen insgesamt für eine frühzeitige chirurgische Intervention an einem spezialisierten Zentrum“, so Höfkens Fazit.

Dr. med. Thomas M. Heim

Quelle: Symposium "Lungenmetastasen und septische Thoraxchirurgie“ am 29.09.2016 im Rahmen der 25. Jahrestagung der Deutschen Gesellschaft für Thoraxchirurgie und 4. D-A-CH-Tagung, Freiburg. 